\title{
Transient Thermal Characteristics of a Heated Infrared Temperature Sensor for Noncontact Medical Thermometry
}

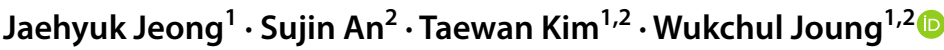

Received: 30 December 2021 / Accepted: 3 February 2022 / Published online: 14 February 2022

(C) The Author(s), under exclusive licence to Springer Science+Business Media, LLC, part of Springer Nature 2022

\begin{abstract}
In this work, the transient responses of a heated infrared (IR) temperature sensor were investigated to improve the reliability of determined target temperatures obtained from IR-based medical thermometers. A medical-grade IR temperature sensor was heated at the lower edge of the sidewall of the sensor. To reduce the uncertainty due to the conversion factor of the thermal detector, the temperature of the target, which was a thermostatted blackbody source, was determined when the observed target temperature and the temperature of the detector coincided during the heating and cooling of the sensor. When the determined target temperature was compared with the blackbody source temperature, it was found that during heating, due to the produced temperature gradient in the sensor, the observed target temperature showed erroneous depressions, resulting in the determined target temperature being considerably lower than the true target temperature. In contrast, the determined target temperature during cooling of the heated sensor was consistent with the tested blackbody source temperatures within the claimed uncertainty at all heating conditions. Therefore, based on the obtained results, it was concluded that temperature measurements using an IR temperature sensor could be carried out with the least uncertainty by determining the target temperature when the observed target and detector temperatures coincided during cooling of the heated sensor.
\end{abstract}

Keywords COVID-19 . Heated thermal detector · Heating location · Heating rate Infrared temperature sensor · Temperature gradient

\section{List of Symbols}

C Calibration constant

Wukchul Joung

wukchuljoung@pknu.ac.kr

1 Department of Intelligent Robot Engineering, Pukyong National University, 45 Yongso-ro, Nam-gu, Busan 48513, Republic of Korea

2 Department of Mechanical Engineering, Pukyong National University, 45 Yongso-ro, Nam-gu, Busan 48513, Republic of Korea 
$g$ Conversion factor

$k$ Coverage factor

$t$ Celsius temperature

$T$ Thermodynamic temperature

$u$ Standard uncertainty

$V \quad$ Electric signal from the thermal detector

\section{Introduction}

The rapid and wide spread of COVID-19 (coronavirus disease-19) has become the most urgent challenge to the public health management system. For effective pandemic containment and proper triage of those infected, early diagnosis of the disease is undeniably important, and detecting fever, one of the most common symptoms of COVID-19, is a widely used method for identifying patients or screening potentially infected persons $[1,2]$. In this context, body temperature measurement using a thermal radiation detector, which determines body temperature based on infrared (IR) radiation from a part of the body, is gaining increasing attention because of its less contagious nature, quick response time, and ease of use.

The current pandemic and the benefits of IR radiation thermometry have led to the widespread use of IR-based medical thermometers, such as infrared ear and skin thermometers, for diagnosing diseases and screening febrile individuals worldwide. However, the unsatisfactory accuracy of IR thermometers has long been an issue in the scientific community [2]. In particular, despite the standard specifications for IR-based thermometers, such as ASTM E1965-98, which specifies that the maximum permissible error be no greater than $0.3{ }^{\circ} \mathrm{C}$ in the $22{ }^{\circ} \mathrm{C}$ to $40{ }^{\circ} \mathrm{C}$ range [3], previous works have shown that many commercially available IR temperature sensors indicate temperatures of calibrated blackbody sources far outside the specified maximum error [4-6], implying that the stated accuracy of IR temperature sensors is hardly reliable and that a method for accurate body temperature measurement at a reduced uncertainty needs to be developed.

IR-based medical thermometry generally uses a thermopile-type thermal detector; it returns an electric signal $(V)$ in response to a temperature difference between the target and the detector. The target temperature $\left(T_{\text {target }}\right)$ is determined by the calibrated dependence of the detector signal on the temperature difference (i.e., by the conversion factor $g$ with a constant $C$, which is a function of the emissivity, geometry, and Stefan-Boltzmann constant) and its own temperature $\left(T_{\text {detector }}\right)$, which is measured by a reference thermometer built in the sensor [7, 8]. Equations 1 and 2 show the expressions for the target temperature obtained from the detector signal and the corresponding uncertainty in the target temperature [7]. Modern integrated circuit fabrication processes have led to the miniaturisation of the thermopile detector, reference thermometer, and control electronics in a variety of structures, 


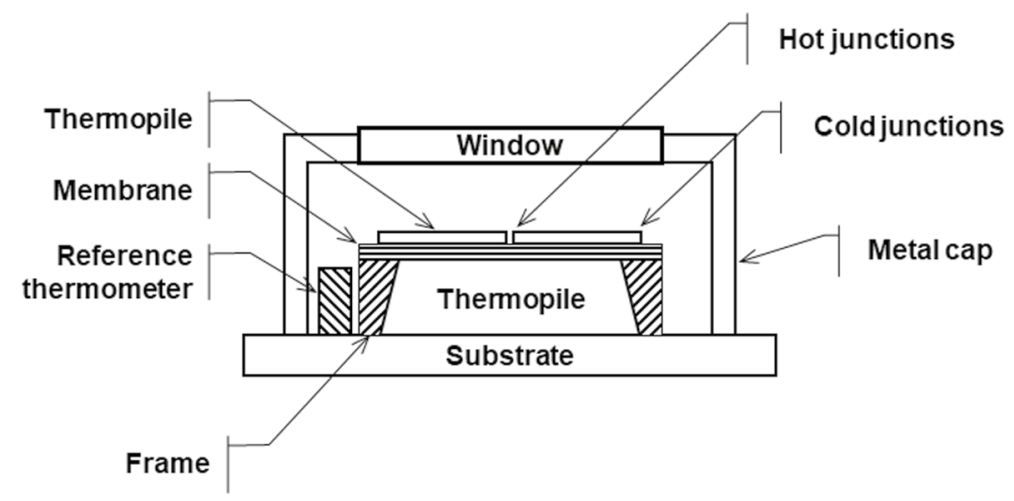

Fig. 1 Structure of the IR-based temperature sensor with a thermopile-type thermal detector

allowing the IR temperature sensor to be built on a single substrate [9]. Figure 1 illustrates the structure of a conventional IR temperature sensor.

$$
\begin{gathered}
T_{\text {target }} \approx T_{\text {detector }}+\frac{V}{C g T_{\text {detector }}^{3}}, \\
u^{2}\left(T_{\text {target }}\right)=u^{2}\left(T_{\text {detector }}\right)+\left(\frac{1}{C g T_{\text {detector }}^{3}}\right)^{2} u^{2}(V)+\left(\frac{V}{C g^{2} T_{\text {detector }}^{3}}\right)^{2} u^{2}(g) .
\end{gathered}
$$

As shown in Eq. 2, three uncertainty factors propagate to the uncertainty of the target temperature; these factors are due to the measurements of the detector temperature and the electric signal $\left(u\left(T_{\text {detectror }}\right)\right.$ and $\left.u(V)\right)$ and the conversion factor $(u(g))$. The contribution (i.e., the sensitivity coefficient) of the uncertainty due to the conversion factor can be reduced in principle by deliberately decreasing the electric signal of the detector $(V)$, which is proportional to the temperature difference between the target and the detector [7], whereas the other two uncertainties are unavoidable. Based on this principle, an IR thermometer with a heated detector is a widely used configuration for reducing the uncertainty of the target temperature by heating the IR temperature sensor (and the detector) to the target temperature (e.g., body temperature), thus removing the detector signal $[7,10,11]$. The detector is heated by a heating element of various configurations and at various locations around the detector; that is, from simple attachment of the heater on the rear, bottom surface of the detector to integration of the heating element on the outer edge of the substrate [11]. In practice, however, during heating, due to the "unwanted" temperature gradient produced in the sensor, a negative signal may be generated, leading to an erroneously low value in the observed target temperature, causing the measured value to be far from the true target temperature [12]. Therefore, a compensation algorithm for this temperature change needs to be employed, which adds unnecessary complications to the operation of the thermometer. 
In this work, to improve the reliability of the target temperature obtained from IR-based medical thermometers, we investigated how heating an IR temperature sensor affects the 'observed' target temperature and proposed a method for determining the true target temperature (i.e., the 'determined' target temperature) at a reduced uncertainty without being influenced by the heating of the sensor. A medical-grade IR temperature sensor was equipped with a heating element at a location found to be optimal based on a numerical heat transfer analysis, and the responses of the IR temperature sensor outputs (i.e., the observed target and detector temperatures) to different thermal transients (i.e., heating and cooling) were monitored while the detector faced a constant temperature blackbody source. The target temperature with the lowest uncertainty, which was the determined target temperature, was then obtained by measuring the detector temperature when the observed target temperature and detector temperature coincided, resulting in a zero sensitivity coefficient to the uncertainty due to the conversion factor. The determined target temperature and the blackbody source temperature were compared to demonstrate the accuracy of the determined target temperature, thus, validating the effectiveness of the suggested method which aimed to ensure accurate target temperature determination while minimising the uncertainty contribution of the conversion factor. The details of the numerical analysis are presented in Sect. 2, the experimental setup and methods are given in Sect. 3, the obtained results and relevant discussions are presented in Sect. 4, and the conclusions are summarised in Sect. 5.

\section{Numerical Analysis}

In this work, the optimal location for heating the IR temperature sensor was determined by numerical heat transfer analysis at various heating locations on the IR temperature sensor. A commercial three-dimensional computational heat transfer analysis tool (ANSYS Fluent) was used to model and analyse the transient temperature distributions under specific heating conditions (e.g., heating location, heating rate, duration of heating). The IR temperature sensor was modelled using simple geometries for efficient computation. Figure $2 \mathrm{a}$ and $\mathrm{b}$ show the modelled sensor and the analysed heating locations, respectively.

The external dimensions and internal layout of the sensor were modelled based on a commercial medical-grade IR temperature sensor (Melexis MLX90614ESFDCA), which is widely used in the manufacture of IR-based medical thermometers. As shown in Fig. 2a, the detector (i.e., the membrane on the frame in Fig. 2a) was located at the centre of the substrate of the sensor, and the reference thermometer that measured the detector temperature was located at a certain distance from the detector. As shown in Fig. 2b, two heating locations on the sidewall of the sensor (labelled as side, upper heating and side, lower heating) and one heating location on the bottom surface of the sensor (labelled as rear, bottom heating) were chosen for calculation based on the heater locations of commercial heated-detector-type IR temperature sensors [11] and a previous work on thermal transient of the sensor 
(a)
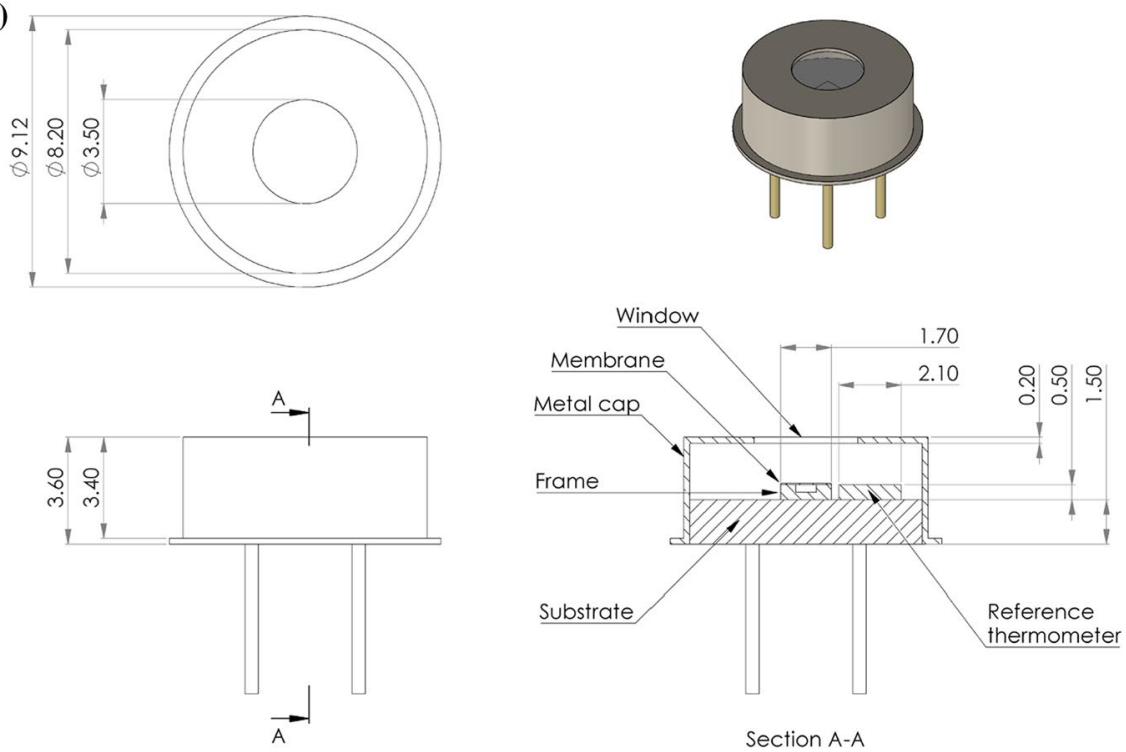

Section A-A

(b)
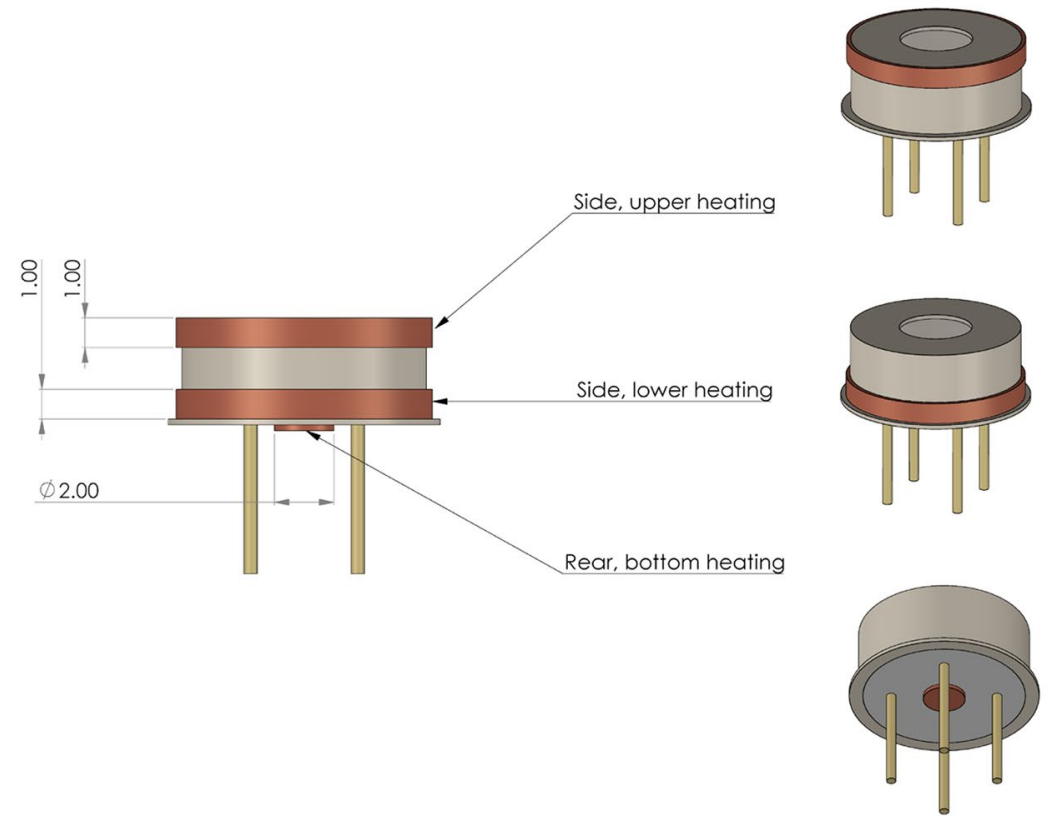

Fig. 2 (a) Simplified model of the IR temperature sensor for the numerical heat transfer analysis. (b) Locations of the heating element compared in the numerical analysis

[12]. For side heating, a ring-type heater with a width of $1 \mathrm{~mm}$ was positioned along the upper edge or lower edge of the sidewall of the sensor; for bottom heating, a disk-shaped heater with a diameter of $2 \mathrm{~mm}$ was attached to the centre of the rear 
bottom surface of the sensor. Due to a lack of information on the material composition of the sensor, the substrate, detector, and reference thermometer were simply modelled as silicon, while the metal cap and window were modelled as stainless steel and glass, respectively.

The heating condition was initially set to a heating rate of $3 \mathrm{~W}$ for a heating duration of $1 \mathrm{~s}$ (i.e., $3 \mathrm{~J}$ ), and the temperature variations at different locations in the sensor from $0 \mathrm{~s}$ to $30 \mathrm{~s}$ were calculated to assess the effectiveness of the tested heating locations in terms of the heating speed. The effect of the "unwanted" temperature gradient, which was due to heating the sensor and is known to cause an erroneous detector signal [12], was investigated by evaluating the temperature difference between the detector (the centre of the frame) and the reference thermometer for each of the tested heating locations. Then, the effect of the heating conditions was studied by varying the heating rate while keeping the total energy of the supplied heat constant (i.e., $3 \mathrm{~W}$ for $1 \mathrm{~s}, 2 \mathrm{~W}$ for $1.5 \mathrm{~s}$, and $1 \mathrm{~W}$ for $3 \mathrm{~s}$ ).

Figure 3 shows the calculated temperature distributions in the IR temperature sensor at different heating locations immediately after heating at $3 \mathrm{~W}$ for $1 \mathrm{~s}$. As shown in the figure, the temperature distributions differed depending on the heating location. In particular, the clearly diverged temperature contour for the rear, bottom heating case and the low substrate temperature for the side, upper heating case demonstrated that these two heating locations were inadequate due to the

(a)

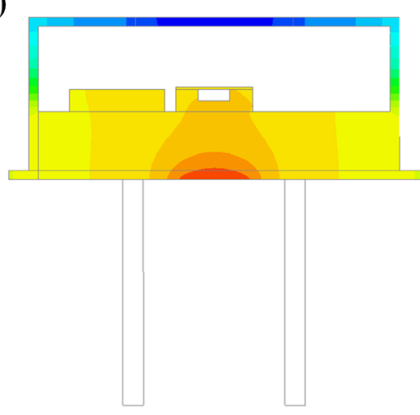

(c)

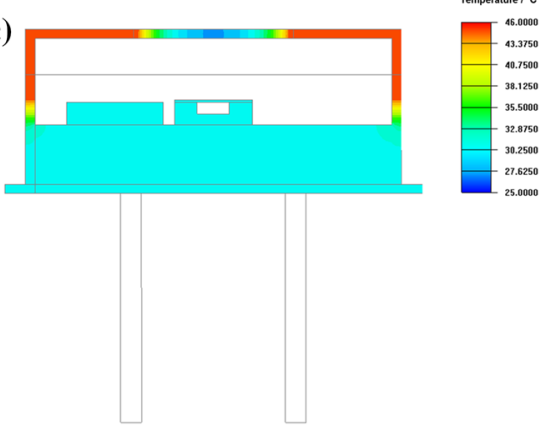

(b)

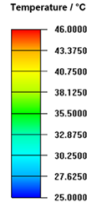

\section{(b)}

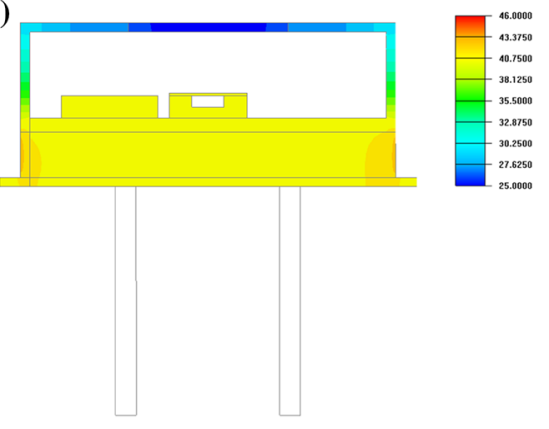

Fig. 3 Calculated temperature distributions in the IR temperature sensor when a heating rate of $3 \mathrm{~W}$ was applied for $1 \mathrm{~s}$ to the tested heating locations. (a) Rear, bottom heating. (b) Side, lower heating. (c) Side, upper heating (Color figure online) 
severe temperature gradient produced in the sensor and the inefficient heating of the detector, respectively. On the other hand, the side, lower heating resulted in a moderately uniform temperature distribution across the substrate (including the detector and the reference thermometer), as well as a relatively high temperature, which was sufficient to raise the detector temperature to the target temperature (e.g., body temperature).

Figures $4 \mathrm{a}$ and $\mathrm{b}$ show the variations in the temperature of the reference thermometer and the temperature difference between the detector and the reference thermometer for different heating locations when heated at $3 \mathrm{~W}$ for $1 \mathrm{~s}$, respectively. As shown in Fig. 4a, the rear, bottom heating and side, lower heating cases were much faster than the side, upper heating case, implying that the side, upper heating case could result in insufficient heating of the detector to reduce the uncertainty contribution of the conversion factor (i.e., the sensitivity coefficient of $u(g)$ in Eq. 2). The variation in the temperature difference between the detector and the reference thermometer in Fig. 4b showed that the rear, bottom heating case could cause a significant error due to the large temperature gradient induced in the sensor during heating. Although the smallest temperature difference was observed in the side, upper heating case, this location was found to be inadequate in terms of the heating speed;

(a)

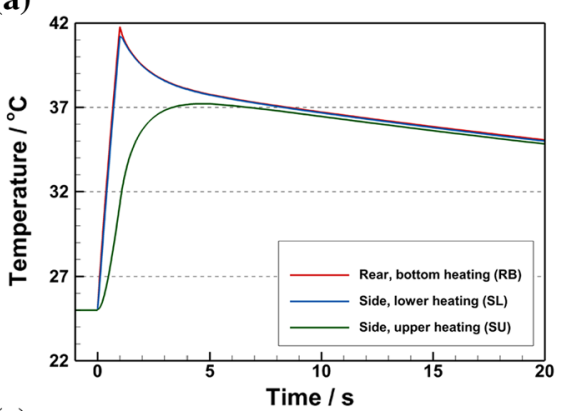

(c)

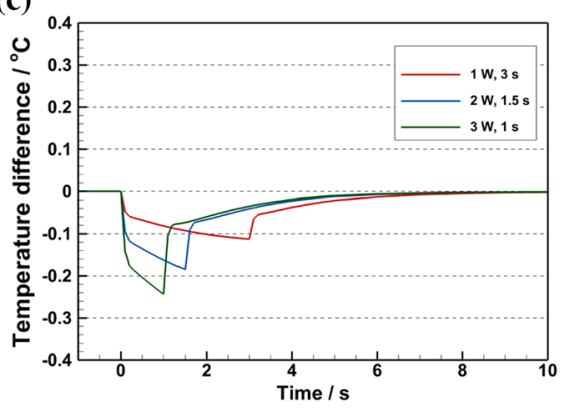

(b)

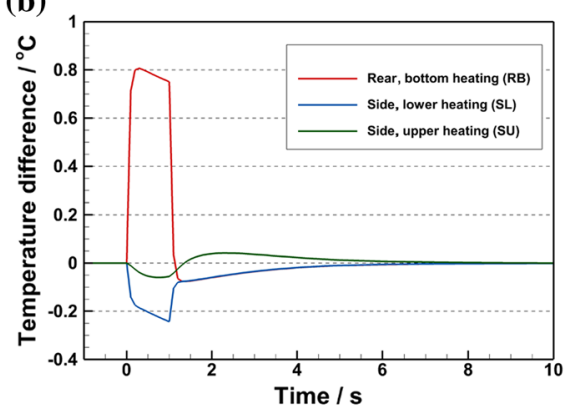

Fig. 4 Results of the transient analyses. (a) Temperature variation of the reference thermometer of the IR temperature sensor at different heating locations when a heating rate of $3 \mathrm{~W}$ was applied for $1 \mathrm{~s}$. (b) Variations in the temperature difference between the frame of the detector and the reference thermometer at different heating locations when a heating rate of $3 \mathrm{~W}$ was applied for $1 \mathrm{~s}$. (c) Variations in the temperature difference between the frame of the detector and the reference thermometer for different heating rates and durations when the sensor was heated from the side, lower heating location (Color figure online) 
thus, the side, lower heating location was determined to be the optimal heating location among the examined heating locations. For all of the heating locations, when the heating was turned off, the temperature difference began to decrease, implying that the induced temperature gradient was being alleviated, and after some time, the temperature difference vanished, indicating that the sensor had recovered to the isothermal condition, although its temperature was still higher than the initial temperature before heating (see the temperature at $7 \mathrm{~s}$ in Fig. 4a).

Figure $4 \mathrm{c}$ shows the change in the temperature difference between the detector and the reference thermometer under various heating conditions for the side, lower heating; the heating rate was changed from $3 \mathrm{~W}$ to $1 \mathrm{~W}$, but the total heating energy remained constant. As illustrated in the figure, as the heating rate decreased, the temperature gradient improved, despite the slower heating. The test results showed that the effect of the increased temperature gradient was to increase the time it took for the sensor to return to its unaffected state; thus, a lower heating rate did not necessarily indicate a slower measurement (this result is discussed further in the later section on the test results). Therefore, in this work, the side, lower heating location was chosen as the optimal heating location for experiments, and different heating conditions (i.e., heating rate and duration) were tested to study the effect of heating on the accuracy of the determined target temperature from the IR temperature sensor.

\section{Experimental Setup and Methods}

In this work, to investigate the transient responses of the IR temperature sensor during heating and cooling under different heating conditions, a commercial IR temperature sensor was equipped with a ring-type heater. The IR temperature sensor was a medical-grade sensor with stated accuracies of $\pm 0.2{ }^{\circ} \mathrm{C}$ from $36{ }^{\circ} \mathrm{C}$ to $38{ }^{\circ} \mathrm{C}$ and $\pm 0.3{ }^{\circ} \mathrm{C}$ from $22{ }^{\circ} \mathrm{C}$ to $40{ }^{\circ} \mathrm{C}$ (excluding the range from $36{ }^{\circ} \mathrm{C}$ to $38{ }^{\circ} \mathrm{C}$ ), in accordance with ASTM E1965-98 [3]; the sensor was operated at $3 \mathrm{~V}$, and the outputs from the sensor (i.e., the observed target and detector temperatures) were obtained via an Arduino communication protocol. The heater was made of a single-wound $\mathrm{Ni}-\mathrm{Cr}$ wire, and it was attached to the lower edge of the sidewall of the sensor, which was determined to be the optimal location based on the numerical analysis. An insulating adhesive film was inserted between the sensor and the heater, and to improve thermal contact, thermal paste was added between the heater and the insulating film. A fixed amount of heating power was supplied to the heater using a feedback-controlled DC power supply for a predetermined heating duration.

The IR temperature sensor was mounted to face a room-temperature blackbody source (Isotech 989) with an emissivity of $0.97 \pm 0.02$, which served as the reference temperature and was used with a calibrated industrial platinum resistance thermometer (IPRT); the calibration uncertainty of the IPRT was $60 \mathrm{mK}(k=2$, approximately $95 \%$ level of confidence), and the resistance of the IPRT was measured using a potentiometer with a stated accuracy of approximately $7 \mathrm{mK}$. The IR temperature sensor was placed along the centreline normal to the blackbody source at a distance of $20 \mathrm{~mm}$ from the source. The temperature stability and uniformity of 
(a)

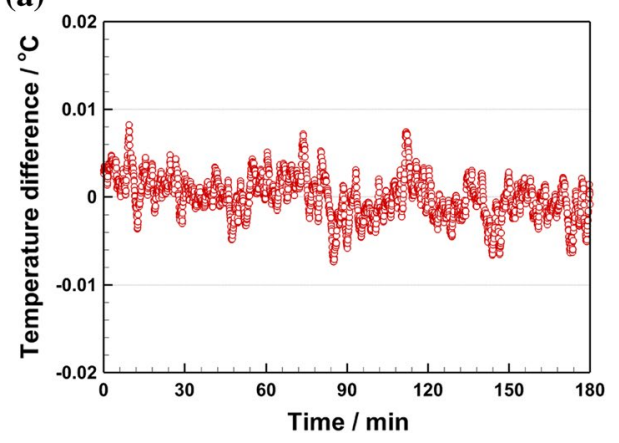

(b)

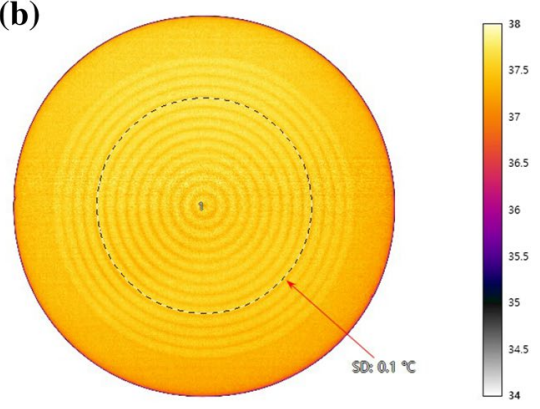

Fig. 5 Temperature stability and uniformity characteristics of the blackbody source (set value: $37^{\circ} \mathrm{C}$ ). (a) Changes in the deviations of the blackbody temperature from the $3 \mathrm{~h}$ mean value. (b) Temperature distribution of the blackbody source measured using a thermographic camera (Color figure online)

the blackbody source were tested to assess the uncertainty of the blackbody source temperature, and the results are shown in Fig. 5a and b. As shown in the figures, the temperature readings from the blackbody source set at $37{ }^{\circ} \mathrm{C}$ were controlled within a standard deviation of approximately $2 \mathrm{mK}$. The temperature distribution of the blackbody source, which was measured with a thermographic camera, revealed that the standard deviation of the temperatures over the circular area with a radius of $20 \mathrm{~mm}$ was approximately $0.1{ }^{\circ} \mathrm{C}$, which corresponded to the field of view (i.e., $90^{\circ}$ ) of the IR temperature sensor at a distance of $20 \mathrm{~mm}$ from the source. The stability and uniformity of the blackbody source temperature was remained similar in the tested temperature range from $34{ }^{\circ} \mathrm{C}$ to $40{ }^{\circ} \mathrm{C}$. Figure 6 shows the schematic of the test setup.

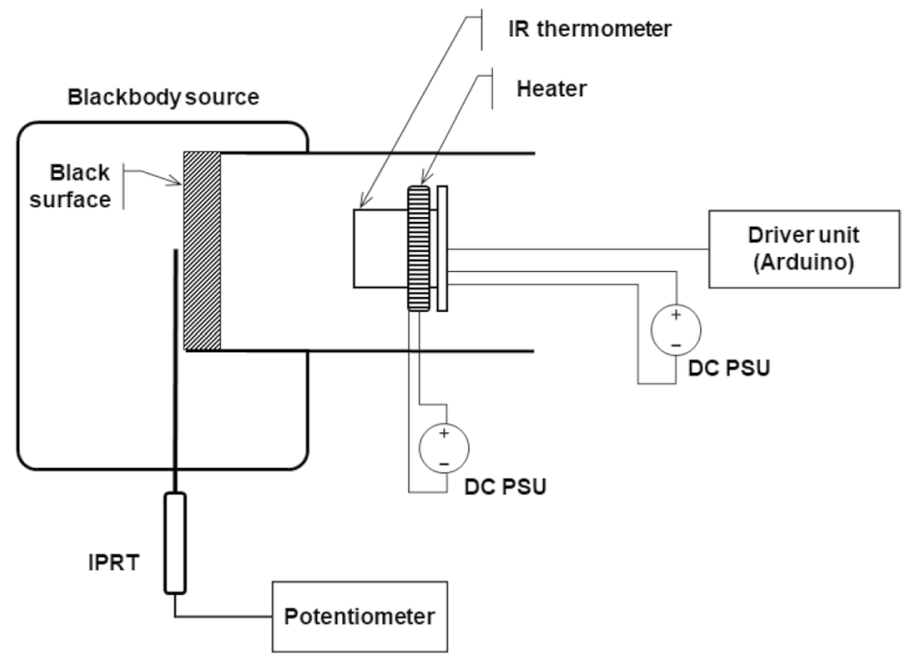

Fig. 6 Schematic of the test setup 
In this study, the transient responses of the IR temperature sensor outputs, which included the observed target and detector temperatures, under different heating conditions (i.e., heating rate and duration) were investigated. The tested heating rates were $1 \mathrm{~W}, 2 \mathrm{~W}$, and $3 \mathrm{~W}$. Unlike the numerical analysis, the heating duration for each heating rate was chosen to be the smallest integral number (in s), which ensured that the detector temperature exceeded the blackbody source temperature and that the temperature difference between the blackbody source and the detector vanished. Thus, when heating at a prescribed heating rate and duration, the temperature of the detector in the IR temperature sensor increased slightly above the blackbody source temperature while passing the observed target temperature, then cooled down to the ambient temperature while passing the observed target temperature again, resulting in two temperature equilibration points (i.e., $T_{\text {target }}=T_{\text {detector }}$ ) during heating and cooling, respectively.

Because the temperature equilibration between the target (the blackbody source) and the detector corresponded to a zero detector signal, minimising the sensitivity coefficient of the uncertainty due to the conversion factor $(V=0$ in Eq. 2$)$, the detector temperatures at these points were chosen to be the 'determined' target temperatures. The determined target temperatures were then compared to the blackbody source temperatures measured by the calibrated IPRT to assess the accuracy of the determined target temperature. The tested blackbody source temperatures were $34{ }^{\circ} \mathrm{C}, 37{ }^{\circ} \mathrm{C}$, and $40{ }^{\circ} \mathrm{C}$, and at each blackbody source temperature, the sensor was heated at different heating rates and for different heating durations. The determined target temperature was measured five times to assess the uncertainty due to repeatability, which was the standard deviation of the mean of the temperature readings (i.e., $t_{\text {blackbody }}$ and $t_{\text {target }}$ ) obtained from the five repeated tests at each tested heating rate, and a rectangular distribution was applied to the stated accuracy of the IR temperature sensor to estimate the uncertainty due to the resolution of the temperature readings from the sensor [4-6].

One additional uncertainty component related to the determined target temperature was the uncertainty of the determination of the temperature equilibration points. In this work, the temperature equilibration point was generally chosen as the point when the difference between the observed target and detector temperatures vanished. However, due to the finite time interval between readings, which was approximately $40 \mathrm{~ms}$ for the tested IR temperature sensor, there could be a temperature mismatch; in this case, the first value when the temperature difference changed its sign was chosen as the equilibrated value. Thus, there should be an uncertainty due to the determination of the temperature equilibration point, which could be assessed as the resolution of the readings (e.g., $0.01{ }^{\circ} \mathrm{C}$ ) when the temperature difference vanished or the temperature difference itself when the temperature difference changed its sign. In this work, an average of these values from five measurements at each heating rate and blackbody source temperature were taken as the uncertainty value. 

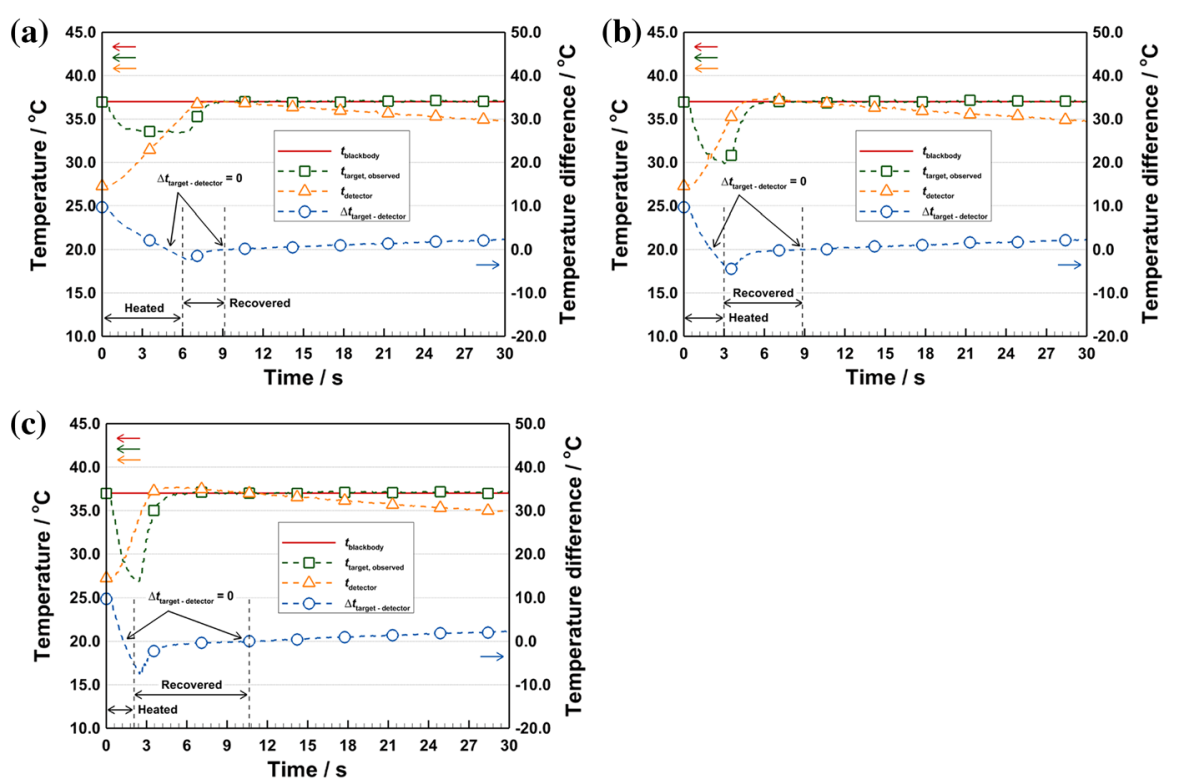

Fig. 7 Transient temperature variations of the target temperature, detector temperature, and the difference between the target and detector temperatures for various heating rates and times. (a) Heated at $1 \mathrm{~W}$ for $6 \mathrm{~s}$. (b) Heated at $2 \mathrm{~W}$ for $3 \mathrm{~s}$. (c) Heated at $3 \mathrm{~W}$ for $2 \mathrm{~s}$

\section{Results and Discussion}

Figure 7a-c show the transient variations of the observed target and detector temperatures $\left(t_{\text {target, observd }}\right.$ and $\left.t_{\text {detector }}\right)$, together with the difference between these two temperatures $\left(\Delta T_{\text {target-detector }}\right)$ and the $37^{\circ} \mathrm{C}$ blackbody source temperature $\left(t_{\text {blackbody }}\right)$ when heated at $1 \mathrm{~W}$ for $6 \mathrm{~s}, 2 \mathrm{~W}$ for $3 \mathrm{~s}$, and $3 \mathrm{~W}$ for $2 \mathrm{~s}$, respectively. As shown in the figures, during heating, the observed target temperature (green square symbol) had spurious depressions despite the constant blackbody source temperature (red solid line). As reported in [12], the temperature depression was caused by a temperature gradient induced by heating the sidewall of the sensor, which generated a negative detector signal against the unaffected signal generated by the temperature difference between the blackbody source and the detector. The magnitude of the temperature depression became more pronounced with increasing heating rate due to the intensified temperature gradient, as predicted by the numerical analysis. Immediately after the heating power was turned off, the observed target temperature began to recover to the unaffected blackbody source temperature as the temperature gradient produced in the sensor was alleviated. During this process, due to the augmented temperature depression with increasing heating rate, the time required for the observed target temperature to recover increased with increasing heating rate.

In contrast to the observed target temperature, the detector temperature increased monotonically during heating, as it resulted from a simple temperature measurement by the reference thermometer mounted on the substrate. When the heater was turned off, the detector temperature continued to increase for some time due to heat transfer 
from the heated (radially) outer region and then began to decrease. The difference between these two temperatures (blue circle symbol) is depicted in Fig. 7. As shown in the figure, there were two temperature equilibration points between the observed target and detector temperatures (i.e., $\Delta t_{\text {target-detector }}=0$ ): the first during the heating of the sensor and the second during the cooling of the heated sensor. Because of the erroneous depression in the observed target temperature during heating, the first resulted in the determined target temperature being far from the true target temperature (i.e., the blackbody source temperature). However, because the second temperature equilibration point occurred after the temperature had recovered, the determined target temperature correctly indicated the blackbody source temperature, at which the uncertainty contribution due to the conversion factor vanished.

Figure $8 \mathrm{a}$ and $\mathrm{b}$ show a comparison of the determined target temperature $\left(t_{\text {target,determined }}\right)$ and the blackbody source temperature (set at $37{ }^{\circ} \mathrm{C}$ ) for different heating rates when the observed target and detector temperatures coincided during heating and cooling of the sensor, respectively. As shown in the figures, during the heating of the sensor, the determined target temperature was far lower than the blackbody source temperature and was outside the claimed uncertainty, indicating that the determined target temperature was incorrect despite the minimal uncertainty condition. This discrepancy was caused by the temperature gradient produced when the sensor was heated, which resulted in depressions in the observed target temperature. Moreover, the deviation of the determined target temperature from the blackbody source temperature was found to increase with increasing heating rate due to the augmented temperature depressions of the observed target temperature at higher heating rates.

In contrast, the determined target temperature during cooling of the heated sensor was consistent with the blackbody source temperature within the claimed uncertainty regardless of the heating rate, indicating that an accurate target temperature determination was possible by measuring the detector temperature when the temperatures equilibration between the observed target and detector temperatures was reached during cooling of the heated sensor. In particular, as the
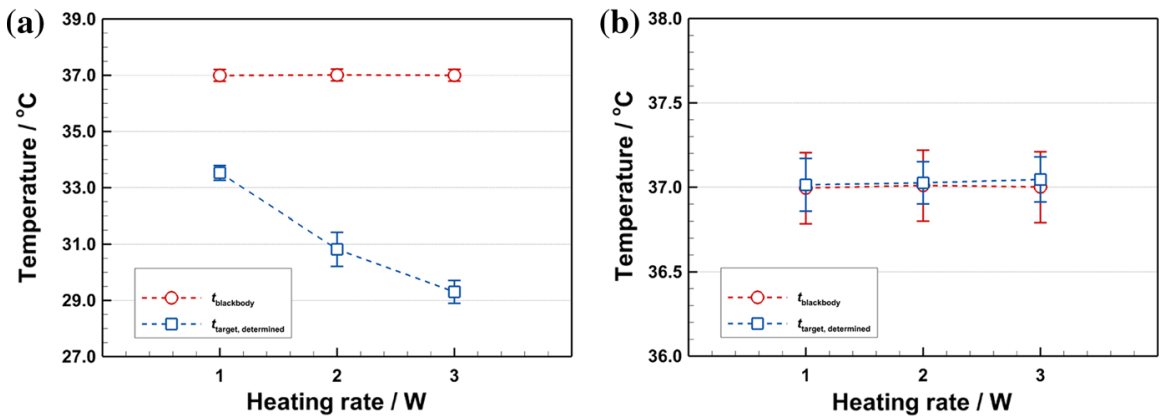

Fig. 8 Comparison of the blackbody temperatures and the target temperatures determined when the observed target and detector temperatures coincided during heating and cooling of the sensor (i.e., $T_{\text {target }}=T_{\text {detector }}$ ). (a) During heating of the detector at $t_{\text {blackbody }}=37^{\circ} \mathrm{C}$. (b) During cooling of the detector at $t_{\text {blackbody }}=37^{\circ} \mathrm{C}$ 


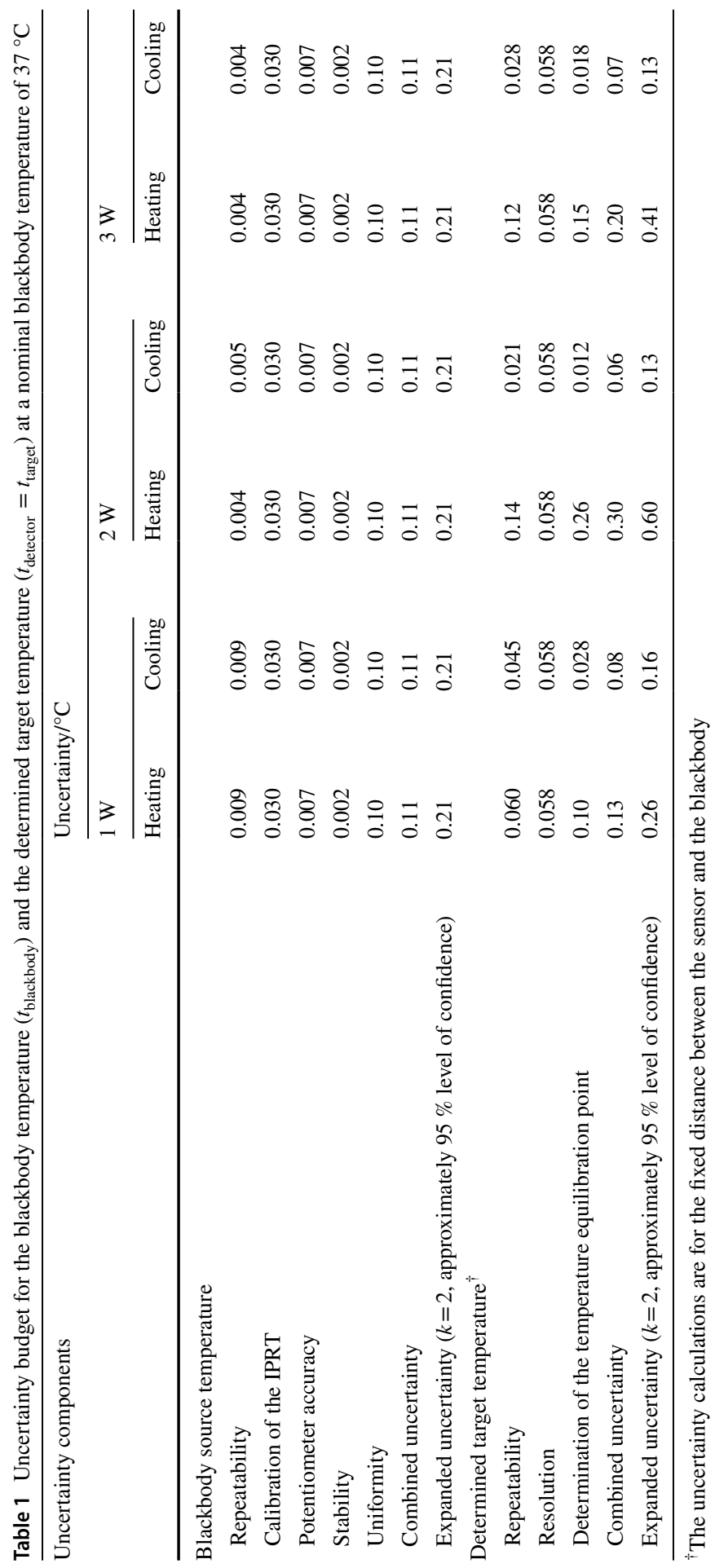


determined target temperature was nearly independent of the heating rate, the temperature gradient produced by heating the sensor was effectively removed as the heated sensor was cooled, ensuring that the target temperature measurement was not influenced by the heating. Table 1 shows the uncertainty budget for the blackbody source temperature and the determined target temperature during heating and cooling of the sensor at a nominal blackbody temperature of $37{ }^{\circ} \mathrm{C}$. Based on these results, it was clear that during heating of the sensor, the temperature difference between the determined target temperature $\left(t_{\text {target }}\right)$ and the true target temperature ( $\left.t_{\text {blakcbody }}\right)$ was outside the claimed uncertainties, whereas the temperature difference during cooling of the heated sensor was within the claimed uncertainties. Therefore, it was found that an accurate target temperature measurement for a heated-type IR temperature sensor could be obtained as the heated sensor cooled but not as the sensor was heated.

In Table 1, the uncertainty of the determined target temperature due to the determination of the temperature equilibration point showed very different values depending on the transient thermal processes which the sensor experienced (i.e., heating or cooling). The uncertainties of the determined target temperature obtained during heating of the sensor was much larger than the uncertainties during cooling of the heated sensor. This behaviour was supposed to be due to the erroneous depression of the observed target temperature during heating of the sensor where the observed target and detector temperatures changed relatively fast; during cooling of the heated sensor, due to the slow changes in temperature, the difference between the observed target and detector temperatures mostly vanished or showed very small values when the sign of the temperature difference changed, thus further supporting the accuracy of the determined target temperature obtained during cooling of the heated sensor.

Figures 9a-d show the results of comparisons between the determined target temperature and the blackbody source temperature (set at $34{ }^{\circ} \mathrm{C}$ and $40{ }^{\circ} \mathrm{C}$ ) during heating and cooling of the sensor. In these cases, due to the larger stated accuracy of the IR temperature sensor (i.e., $0.3{ }^{\circ} \mathrm{C}$ ), which affected the resolution of the detector temperature, the uncertainty of the determined target temperature was slightly increased compared to the blackbody source temperature of $37^{\circ} \mathrm{C}$. Tables 2 and 3 show the uncertainty budgets for the blackbody source temperature and the determined target temperature during heating and cooling of the sensor at nominal blackbody temperatures of $34{ }^{\circ} \mathrm{C}$ and $40{ }^{\circ} \mathrm{C}$, respectively. As shown in the figures, similar to the previous case of the blackbody source at $37{ }^{\circ} \mathrm{C}$, the deviations in the determined target temperatures at the temperature equilibration point during sensor heating were larger than the claimed uncertainty from the tested blackbody source temperatures at all tested heating rates.

On the other hand, the determined target temperature during cooling of the heated sensor was consistent with the tested blackbody source temperatures within the claimed uncertainty at all tested heating rates, as it was unaffected by the heating of the sensor, thus proving the correctness and validity of the target temperature determination method at the smallest uncertainty contribution of the conversion factor. Overall, the results showed that temperature measurements using an IR temperature sensor could be carried out with the smallest uncertainty contribution of the conversion factor by determining the target 

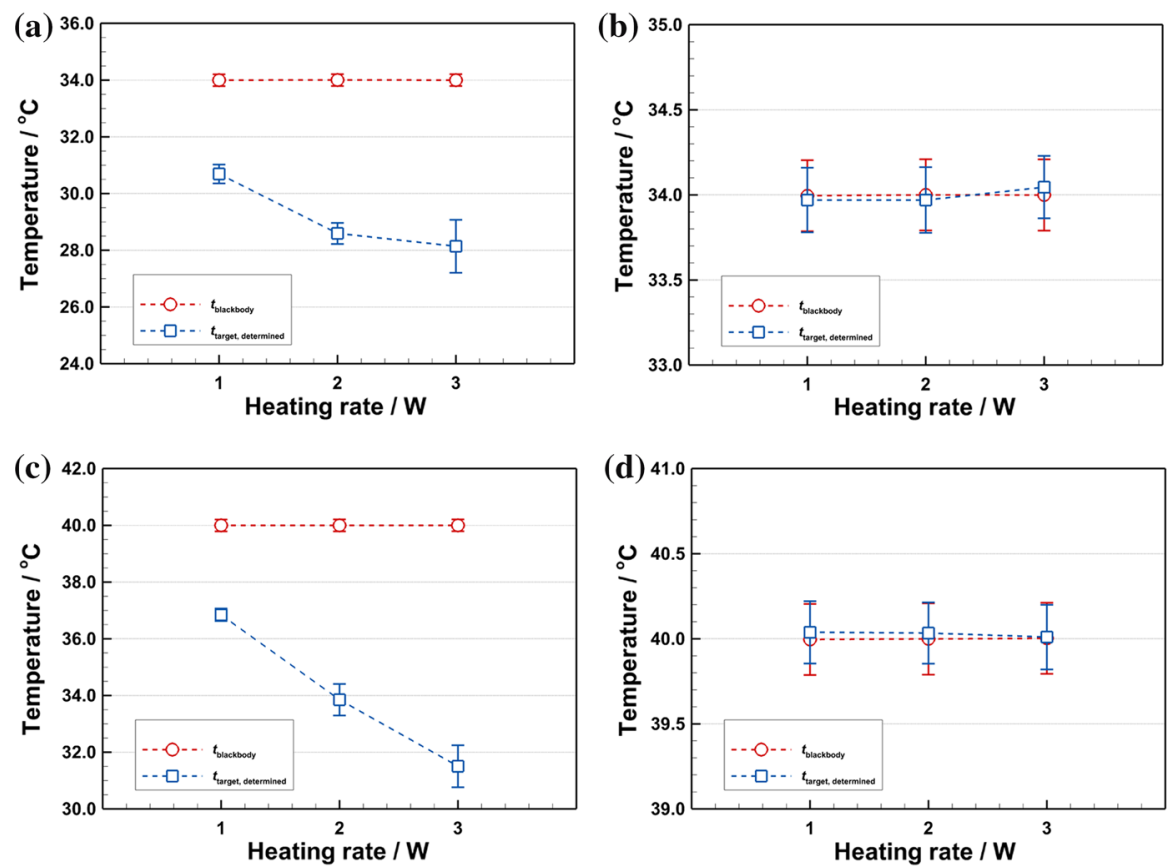

Fig. 9 Comparison of the blackbody temperatures and the determined target temperatures when the observed target and detector temperatures coincided during heating and cooling of the sensor (i.e., $T_{\text {target }}=T_{\text {detector }}$ ). (a) During heating of the detector at $t_{\text {blackbody }}=34^{\circ} \mathrm{C}$. (b) During cooling of the detector at $t_{\text {blackbody }}=34{ }^{\circ} \mathrm{C}$. (c) During heating of the detector at $t_{\text {blackbody }}=40^{\circ} \mathrm{C}$. (d) During cooling of the detector at $t_{\text {blackbody }}=40^{\circ} \mathrm{C}$

temperature when the two sensor temperature readings (i.e., the observed target and detector temperatures) coincided during cooling of the heated sensor. One limitation of this finding is the prior knowledge of the target temperature, based on which the heating rate and duration were determined. In practice, the suggested algorithm could be implemented in a stepwise manner; for example, first determining appropriate heating rate and duration based on an approximate 'observed target temperature' without heating, which is attained instantly, and then heating the sensor to measure the accurate 'determined target temperature' during cooling of the heated sensor. In this case, a further study for a fully automated algorithm determining the target temperature is necessary.

Although the validity of the suggested method was proven by the comparison results, for practical use of IR temperature sensors, the time required for the sensor to recover from the induced temperature gradient should be further optimised by adjusting the heating rate and duration to minimise the measurement time. In addition, as the heating element used in this study was manually fabricated and attached to the sidewall of the sensor, the heat transfer to the detector was less efficient. As a result, for a blackbody source temperature of $37^{\circ} \mathrm{C}$, the 


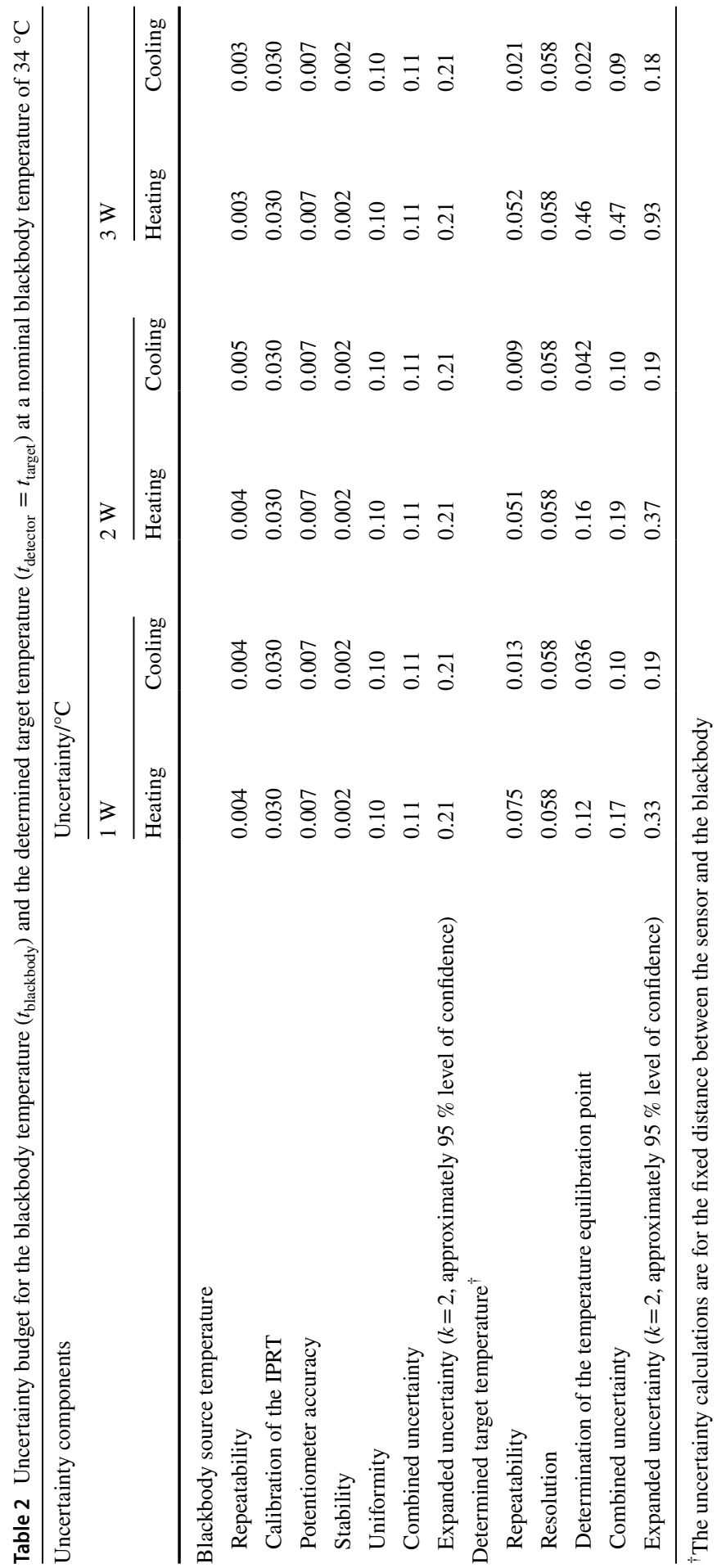




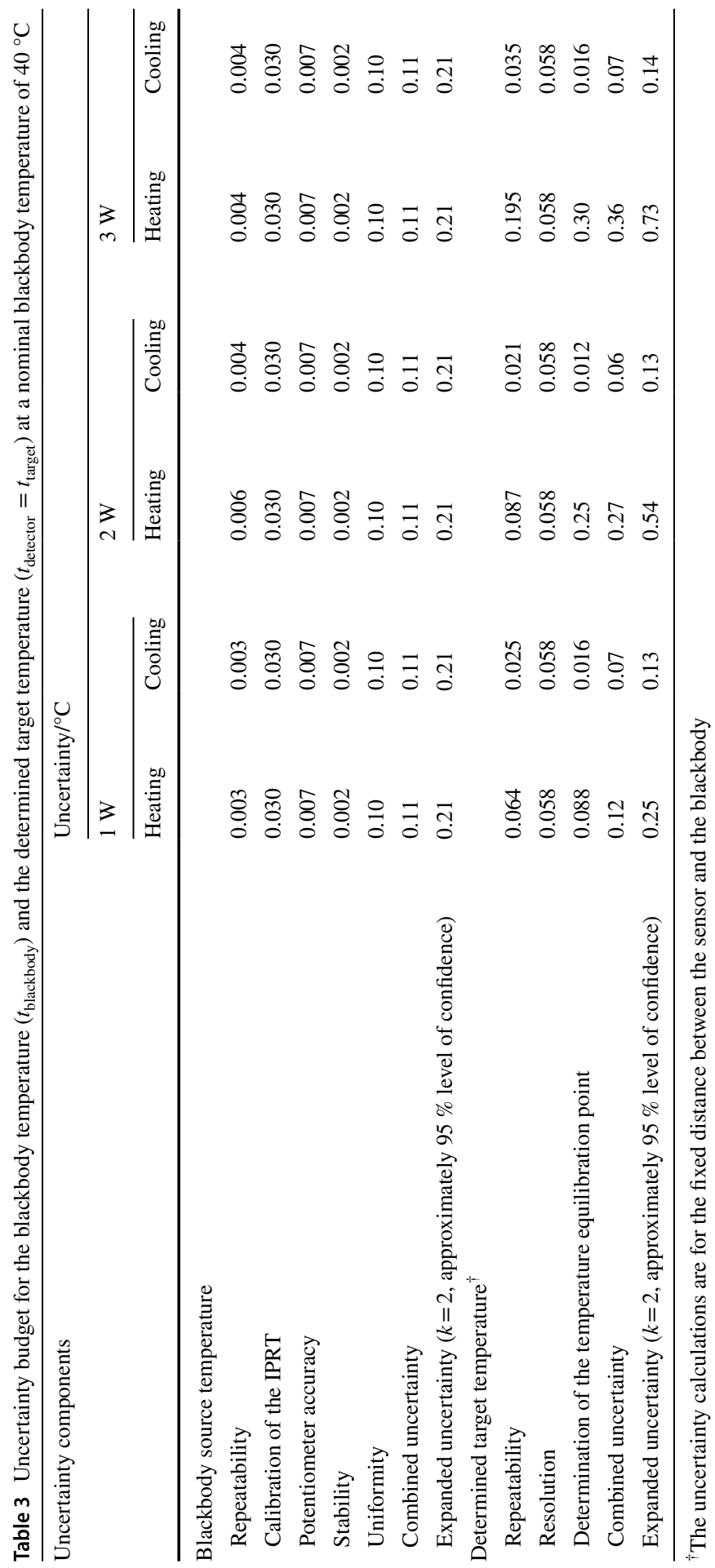


time required to determine the target temperature (including the heating duration) was approximately $10 \mathrm{~s}$, which was considerably longer than the standard measurement time for IR medical thermometry. Thus, to improve the measurement speed and accuracy, an integrated heating element in the IR temperature sensor, which could heat the detector efficiently with a minimum temperature gradient, should be used to simultaneously reduce the measurement uncertainty and time.

\section{Conclusions}

In this work, the transient responses of a heated IR temperature sensor were investigated under different heating conditions to improve the accuracy of the determined target temperature from IR-based medical thermometers. A medicalgrade IR temperature sensor was equipped with a ring-type heater at the lower edge of the sidewall of the sensor, which was found to be the optimal location in terms of heating speed and induced temperature gradient based on a numerical analysis. To reduce the uncertainty contribution of the conversion factor of the thermal detector, the temperature of the target, which was a blackbody source at a predetermined temperature, was determined when the two temperature readings from the sensor (i.e., the observed target and detector temperatures) coincided during heating and cooling of the sensor, resulting in two determined target temperatures at two temperature equilibration points during heating and cooling. Then, the accuracy of the determined target temperatures was assessed by comparing the determined temperatures with the blackbody source temperature.

According to the test results, as the sensor was heated, due to the temperature gradient produced in the sensor, the observed target temperature showed erroneous depressions despite the constant true target temperature (i.e., the blackbody source temperature), resulting in an inaccurate determined target temperature at the first temperature equilibration point during heating. Moreover, the deviation of the determined target temperature from the blackbody source temperature increased with increasing heating rate, indicating that the determined temperature had a strong dependence on the temperature gradient induced in the sensor. On the other hand, the determined target temperature at the second temperature equilibration point, during cooling of the heated sensor, was consistent with the tested blackbody source temperatures within the claimed uncertainty at all tested heating rates, being independent of the heating rate. Therefore, based on the obtained results, it was concluded that temperature measurements using an IR temperature sensor could be carried out with the least uncertainty contribution of the conversion factor by determining the target temperature when the observed target and detector temperatures coincided during cooling of the heated sensor.

Acknowledgments This work was supported by a Grant from the Korea Health Technology R\&D Project through the Korea Health Industry Development Institute (KHIDI), funded by the Ministry of Health \& Welfare, Republic of Korea (HW20C2170020020).

Data Availability All the raw data is available (if requested). 
Code Availability Not applicable.

\section{Declarations}

Conflict of interest The authors declare that there are no known conflict of interest or personal relationships that could have appeared to influence the work reported in this paper.

\section{References}

1. G. Machin et al., J. Med. Eng. Technol. 45, 136 (2021). https://doi.org/10.1080/03091902.2021. 1873441

2. G. Machin et al., Thermol. Int. 31, 5 (2021)

3. ASTM E1965-98(2009), Standard Specification for Infrared Thermometers for Intermittent Determination of Patient Temperature (ASTM International, West Conshohocken, 2009)

4. I. Pusnik, E. van der Ham, J. Drnovsek, Physiol. Meas. 25, 69 (2004). https://doi.org/10.1088/0967$3334 / 25 / 3 / 010$

5. I. Pusnik, J. Drnovsek, Physiol. Meas. 26, 1075 (2005). https://doi.org/10.1088/0967-3334/26/6/016

6. T. Fetcher, A. Whittam, R. Simpson, G. Machin, J. Med. Eng. Technol. 42, 65 (2018). https://doi. org/10.1080/03091902.2017.1409818

7. J.G. Webster, H. Eren (eds.), Measurement, Instrumentation, and Sensors Handbook, 2nd edn. (CRC Press, Boca Raton, 2014), p. 72

8. A. Graf, M. Arndt, M. Sauer, G. Gerlach, Meas. Sci. Technol. 18, R59 (2007). https://doi.org/10. 1088/0957-0233/18/7/R01

9. J. Schieferdecker, R. Quad, E. Holzenkampfer, M. Schulze, Sens. Actuator A Phys. 47, 422 (1995). https://doi.org/10.1016/0924-4247(94)00934-A

10. B. Kraus, A. Klos, US patent, No. 7014358 (2006)

11. B. Kraus, European patent, No. 1081475 (2005)

12. M. Liess, H. Karagozoglu, H. Ernst, Sens. Actuator A Phys. 154, 1 (2009). https://doi.org/10.1016/j. sna.2009.05.013

Publisher's Note Springer Nature remains neutral with regard to jurisdictional claims in published maps and institutional affiliations. 